\section{Drop the language of disorder}

\section{INTRODUCTION}

We may be on the cusp of a major paradigm shift in our thinking about psychiatric disorders. The proposed revision of the American Psychiatric Association's Diagnostic and Statistical Manual (DSM) of Mental Disorders franchise for the classification and diagnosis of human distress, which will lead to the 5th edition (DSM-V), has served as a catalyst for a wide range of criticism (most notably at www.ipetitions.com/petition/dsm5/). This has identified serious inadequacies in the specific proposed revisions, and has also highlighted scientific, philosophical, practical and humanitarian weaknesses in the diagnostic approach to psychological well-being, underpinning the DSM. This debate provides the opportunity to propose a more scientific grounded and clinically useful system.

\section{PROBLEMS WITH DIAGNOSIS}

Diagnostic systems in psychiatry have always been criticised for their poor reliability, validity, utility, epistemology and humanity. With great effort, and standardised approaches, it is possible for reliable diagnoses to be generated. But such practices are rarely adopted in clinical settings, and as we know, it is entirely possible to reliably diagnose invalid diagnoses (the mere agreement between diagnosticians is no guarantee that diagnoses correspond to meaningful clusters of symptoms, with distinct pathophysiology and aetiology, which predict the effectiveness of particular treatments).

The poor validity of psychiatric diagnoses-their inability to map onto any entity discernable in the real world-is demonstrated by their failure to predict course or indicate which treatment options are beneficial, and by the fact that they do not map neatly onto biological findings, which are often nonspecific and cross diagnostic boundaries. For example, depression and anxiety disorders are so comorbid that it is often arbitrary which diagnosis is given to a patient; schizophrenia symptoms are usually accompanied by mood symptoms and overlap with those of bipolar disorder, and it is unclear as to whether bipolar disorder is distinct from major depression.

In epistemological terms, diagnoses convey the idea that people's difficulties can be understood in the same way as bodily diseases, which excludes the possibility of finding meaning in people's 'disordered' responses and experiences, and thus prevents people from understanding how they might use their own resources to address their difficulties. Worse still, diagnoses are used as pseudo-explanations for troubling behaviours (he did this because he has schizophrenia) without consideration of the circularity of that argument, and the broader context (eg, whether a paranoid person has actually been victimised). And as a result of all these failings, the diagnostic tools that we are currently living with mean a person's social and interpersonal difficulties are often ignored in the hope that the right medication regimen will achieve the desired return to normal functioning.

If implemented, the DSM-V would lead to a lowering of a swathe of diagnostic thresholds. This would inflate the assumed prevalence of mental health problems in the general population. This might be good news for pharmaceutical companies, but is a potential threat to the general public and especially vulnerable populations such as children and older people. The clients and the general public are negatively affected by the continued and continuous medicalisation of their understandable responses to their experiences; responses that undoubtedly have distressing consequences which demand helping responses, but which are better understood as normal individual variation than as illnesses.

DSM-V would, if implemented, see an increased emphasis on the supposed biological underpinnings of psychological distress, in that the language of biological illness will be reinforced. This is again concerning, since most scientific evidence points to the fact that complex, individual, interactions between biological, social and psychological factors lead to these distressing experiences.

Finally, such approaches, by introducing the language of 'disorder', undermine a humane response by implying that these experiences indicate an underlying defect. We have seen significant opposition to the proposal that grief, in essence, be pathologised, ${ }^{1}$ but the pathologising of normality in DSM-V is more pervasive, and is shared in all medical diagnostic systems. The death of a loved one can lead to a profound, and long-lasting, grieving process. War is hell. In what sense is it a 'disorder' if we remain distressed by bereavement after 3 months or if we are traumatised by the experience of industrialised military conflict? It is important for all of us to ensure that our children learn appropriately to regulate their emotions and grow up with a sense of moral and social responsibility. But is it appropriate to invoke the concept of 'disorder' when children need extra help?

\section{AN ALTERNATIVE}

We need a wholesale revision of the way we think about psychological distress. We should start by acknowledging that such distress is a normal, not abnormal, part of human life-that humans respond to difficult circumstances by becoming distressed. Any system for identifying, describing and responding to distress should use language and processes that reflect this position. We should then recognise the overwhelming evidence that psychiatric symptoms lie on continua with less unusual and distressing mental states. There is no easy 'cut-off' between 'normal' experience and 'disorder'. We should also recognise that psychosocial factors such as poverty, unemployment and trauma are the most strongly evidenced causal factors for psychological distress ${ }^{2}$ although, of course, we must also acknowledge that other factors-for example, genetic and developmental-may influence the magnitude of the individual's reaction to these kinds of circumstances.

There are alternative systems for identifying and describing psychological distress that may be helpful for the purposes of clinical practice, communication, record-keeping, planning and research, such as the operational definition of specific experiences or phenomena. Some international effort will be needed to develop a shared lexicon, but it is relatively straightforward to generate a simple list of problems that can be reliably and validly defined; for example, depressed mood, auditory hallucinations and intrusive thoughts. There is no reason to assume that these phenomena cluster into discrete categories or other simple taxonomic structure. Indeed, the extent to which the phenomena co-occur may be a function of development and social circumstances. As with many other areas of medicine (particularly primary care) and wider civil society, such problems lend themselves to communication between professionals and the planning of services, especially if it is recognised that the operational definition should include some measure of severity.

While some people find a name or a diagnostic label helpful, our contention is that this helpfulness results from a 
knowledge that their problems are recognised (in both senses of the word), understood, validated, explained (and explicable) and have some relief. Clients often, unfortunately, find that diagnosis offers only a spurious promise of such benefits. Since, for example, two people with a diagnosis of 'schizophrenia' or 'personality disorder' may possess no two symptoms in common, it is difficult to see what communicative benefit is served by using these diagnoses. Surely a description of a person's real problems would suffice? A description of an individual's actual problems would provide more information and be of greater communicative value than a diagnostic label.

For clinicians, working in multidisciplinary teams, the most useful approach would be to develop individual formulations; consisting of a summary of an individual's problems and circumstances, hypothesis about their origins and possible therapeutic solutions. This 'problem definition, formulation' approach rather than a 'diagnosis, treatment' approach would yield all the benefits of the current approach without its many inadequacies and dangers. It would require all cliniciansdoctors, nurses and other professionals - to adopt new ways of thinking. It would also require the rewriting of most standard textbooks in psychopathology (which typically use DSM diagnoses as chapter headings).

For researchers, trying to understand the causes of, and proper responses to, such distress is actively hampered by the diagnostic systems currently used. Whether we are pursuing biological, psychological or social causes of human distress, an invalid diagnostic system is an active hindrance-if there is no validity to a label such as 'schizophrenia', how can researchers finds its cause? Researchers would be better advised to study the nature of, causes of and proper response to specific, identified problems. Indeed, this process has already begun, with a rich literature on social origins, biological substrate and consequences of particular psychiatric phenomena (eg, hallucinations, paranoid delusions and thought disorder) emerging over the last 20 years.

Clinicians are also likely to be more effective if they respond to an individual's particular difficulties rather than their diagnostic label.

Peter Kinderman, ${ }^{1}$ John Read, ${ }^{2}$ Joanna Moncrieff, ${ }^{3}$ Richard P Bentall ${ }^{1}$ ${ }^{1}$ Institute of Psychology, Health and Society, University of Liverpool, Liverpool, UK

${ }^{2}$ Psychology Department, University of Auckland, Auckland, New Zealand

${ }^{3}$ Mental Health Sciences Unit, University College London, London, UK

Correspondence to Professor Peter Kinderman, Institute of Psychology, Health and Society, University of Liverpool, Liverpool L69 3GL, UK; p.kinderman@liverpool.ac.uk

Competing interests None.

\section{REFERENCES}

1. Living with grief. Lancet (Editorial). 2012;379:589.

2. Read J, Bentall RP. Negative childhood experiences and mental health: theoretical, clinical and primary prevention implications. Br J Psychiatry 2012;200:89-91. 